\title{
Is There a Higher Incidence of Sporadic Renal Angiomyolipoma in Childhood Cancer Survivors?
}

\author{
Jarmila Kruseová (D) \\ Barbora Gottfriedová ${ }^{2}$ \\ Andrea Zichová \\ Karel Švojgr' \\ Petr Hošek $\mathbb{1 D}^{3}$ \\ Aleš Lukšl \\ Martin Kynčl ${ }^{2}$ \\ Tomáš Eckschlager \\ 'Department of Paediatric Haematology \\ and Oncology, 2nd Faculty of Medicine, \\ Charles University and Motol University \\ Hospital, Prague, Czech Republic; \\ ${ }^{2}$ Department of Radiology, 2nd Faculty of \\ Medicine, Charles University and Motol \\ University Hospital, Prague, Czech \\ Republic; ${ }^{3}$ Biomedical Center, Faculty of \\ Medicine in Pilsen, Charles University, \\ Pilsen, Czech Republic
}

\begin{abstract}
Background: Cancer treatment can cause various long-term side effects, including those that impact ultrasound findings. During follow-up of childhood cancer survivors (CCSs), we often detected sporadic renal angiomyolipomas without histological confirmation (SAMLs), which is why we initiated this study. We compared the occurrence of SAML in CCSs to the previously reported data from a non-cancer population and correlated SAML with cancer treatment-related factors.
\end{abstract}

Methods: The cohort included 1098 CCSs (median age at cancer diagnosis (dg) 4.3 years) who had ultrasound follow-up (2014-2019). Of the CCSs, 525 (48\%) were female, 132 $(12 \%)$ had subsequent neoplasms (SNs), and 110 (10\%) had genetic syndromes. CCSs were treated for lymphomas $269(24 \%)$ and solid tumors 829 (76\%). None of the CCSs had tuberous sclerosis complex (TSC).

Results: SAML developed in 48 (4.4\%) CCSs; of these, $20(42 \%)$ had SNs. The coincidence of SAMLs and SNs was found in CCSs with a follow-up period exceeding 20 years. The median age at SAML dg was 27.9 years (interquartile range (IQR) 22.3-34.1), and the median time to SAML dg was 22.6 years (IQR 17.4-27.6). Twenty-one (44\%) CCSs developed multiple or bilateral SAMLs lesions; of these, six $(12 \%)$ were in the radiotherapy field. SAML occurrence correlated with radiotherapy of the retroperitoneum (1.65-fold higher with $95 \%$ CI $0.90-3.02$ ). The correlations with other cancer treatment factors and with female sex were less clear.

Conclusion: This study revealed the occurrence of SAMLs in CCSs to be 10 times higher than that in non-cancer studies. The current characteristics of CCSs with SAMLs: younger age, and more bilateral or multiple lesions are more similar to TSC associated angiomyolipoma. Moreover, we observed a coincidence of SAMLs with SNs. Our results support the hypothesis that SAML development in CCSs is not simply a late effect of therapy, and indicates other factors are involved in SAML development.

Keywords: cancer survivorship, angiomyolipoma, ultrasonography, pediatric, surveillance

\section{Plain Language Summary}

We have frequently detected sporadic renal angiomyolipomas (SAMLs) during routine followup ultrasound testing of childhood cancer survivors. As there are no published studies of the frequency for the development and risk factors for SAMLs in childhood cancer survivors, we started this novel study. We observed that the occurrence of SAMLs in childhood cancer survivors is at least 10 times higher than the rate reported in non-cancer studies. Compared to the general population childhood cancer survivors with SAMLs were younger and had more multiple or bilateral lesions. The current characteristics of SAMLs in childhood cancer survivors has similar features with angiomyolipomas associated with genetic syndrome - tuberous sclerosis complex. In addition, over a longer period of follow-up we observed a coincidence of SAML
Correspondence: Jarmila Kruseová Department of Paediatric Haematology and Oncology, 2nd Faculty of Medicine, Charles University and Motol University Hospital, V Uvalu 84/I, 150 06, Prague, 5 Czech Republic

Tel +420601 376302

Fax +420224436420

Email jarmila.kruseova@fnmotol.cz 
with subsequent neoplasm development. For these reasons, we recommend different yearly continuous surveillance of childhood cancer survivors who develop SAMLs comparing to less frequent follow-up of SAMLs in general population.

\section{Introduction}

Curative therapies for cancer can cause various late effects, which may lead to abnormal appearances on subsequent follow-up surveillance imaging, e.q. changes of parenchymal tissue (steatosis, fibrosis, atrophy), and subsequent benign, or malignant neoplasms. During standard ultrasound follow-up of our childhood cancer survivors (CCSs), sporadic renal angiomyolipomas without histological confirmation (SAMLs) were consistently detected. SAMLs are uncommon benign tumors most often affecting the kidney composed of blood vessels, smooth muscles, and adipose tissue in varying proportions ${ }^{1-3}$ and are incidentally detected in older people by diagnostic imaging performed for various medical reasons. ${ }^{4,5}$ They usually present as unilateral single lesions. ${ }^{1-3}$ The prevalence in the general population is reported to be $0.1-$ $0.44 \%$. ${ }^{4,5}$ Previous studies in non-cancer populations reported a higher SAML prevalence in women, and the effect of sex hormones was considered. ${ }^{6}$

Literature regarding angiomyolipomas in younger people is scarce, but an association with tuberous sclerosis complex (TSC) has been identified. ${ }^{1,3}$ For this genetic syndrome (GS) multiple and bilateral lesions and no female predominance are typical. ${ }^{2,3,7}$ Angiomyolipomas occurs in $55-90 \%$ of TSC patients. ${ }^{3,4}$ TSC is caused by inactivating heterozygous or mosaic mutations in TSC2 $(\sim 4 / 5)$ or TSC1 gene $(\sim 1 / 5),{ }^{8,9}$ and angiomyolipomas in TSC patients have a biallelic inactivation of either the TSC2 or TSC1 gene. $^{8-10}$ Although according to noncancer studies ${ }^{3,7,11}$ the etiology of SAMLs is unknown, Giannikou et $\mathrm{al}^{8}$ and Henske et $\mathrm{al}^{10}$ detected somatic biallelic TSC2 inactivation in patients with SAMLs without TSC.

There are no published studies of the frequency and nature of and risk factors for the development of SAMLs in CCSs, except for a few case reports. ${ }^{12,13}$ We have frequently detected SAML during ultrasound analysis, and moreover, we observed a coincidence of SAML with various subsequent neoplasms (SNs) development. Confirmation of the SAML diagnosis usually requires repeated ultrasound analysis, or MRI examinations, which is a psychological burden for the survivor and family, and is a cost burden to the health system. That is why we initially dealt with this issue. The first aim of this study was to describe the occurrence of SAML in CCSs and discuss it within the context SAML incidence previously reported for "non-cancer populations". The second aim was to correlate SAMLs with cancer treatment-related factors.

\section{Patients and Methods}

We analyzed 1098 CCSs who underwent abdominal ultrasound scans during follow-up from 2014 to 2019. All CCSs were $<19$ years old at the time of first cancer diagnosis, and were treated at the Department of Paediatric Haematology and Oncology, Motol University Hospital, Prague, Czech Republic. None had SAMLs at the time of first cancer diagnosis. Of the analyzed CCSs, 525 (48\%) were female, 132 (12\%) developed subsequent neoplasms (SNs) during follow-up, and 110 (10\%) had GSs. A cohort definition is provided in Figure 1. The CCSs had the following GS: Rb-1 deletion (Rb-1del), 28; Neurofibromatosis type 1 (NF1), 18; Familial adenomatous polyposis (FAP), 7; Li-Fraumeni syndrome (LFS), 6; Beckwith-Wiedemann syndrome (BWS), 5; Multiple Endocrine Neoplasia syndrome type 1 (MEN1), 5; and other infrequent GSs (each identified in $\leq 5 \mathrm{CCS}$ ), 41. None of the CCSs had TSC. The CCSs with SNs included 70 CCSs who had at least one subsequent malignant neoplasm (SMN) and 62 CCSs who had at least one histologically confirmed subsequent benign neoplasm (SBN).

The ultrasound assessments were part of standard follow-up care after the end of cancer treatment. CCSs underwent screening for tumor recurrences first five years according to the treatment protocol guidelines, and then CCSs participated in the institutional Motol Late Effect Studies (MLES) for the early detection of SN development. The SAMLs and correlations with cancer treatmentrelated factors, GSs, and $\mathrm{SNs}$ were analyzed. More detailed information about the CCSs is provided in Table 1.

\section{Ultrasound Assessment}

The classic ultrasound appearance of SAMLs is well defined a markedly hyperechoic mass relative to normal renal parenchyma with acoustic shadowing, but no calcification. ${ }^{1-3}$ The ultrasound images were analyzed by two experienced radiologists. They confirmed each SAML ultrasound finding via the hospital database. The number, size, and progression $\geq 3 \mathrm{~mm}$ in the largest dimension of the SAMLs in multiple ultrasound assessments were analyzed. The date of the first 


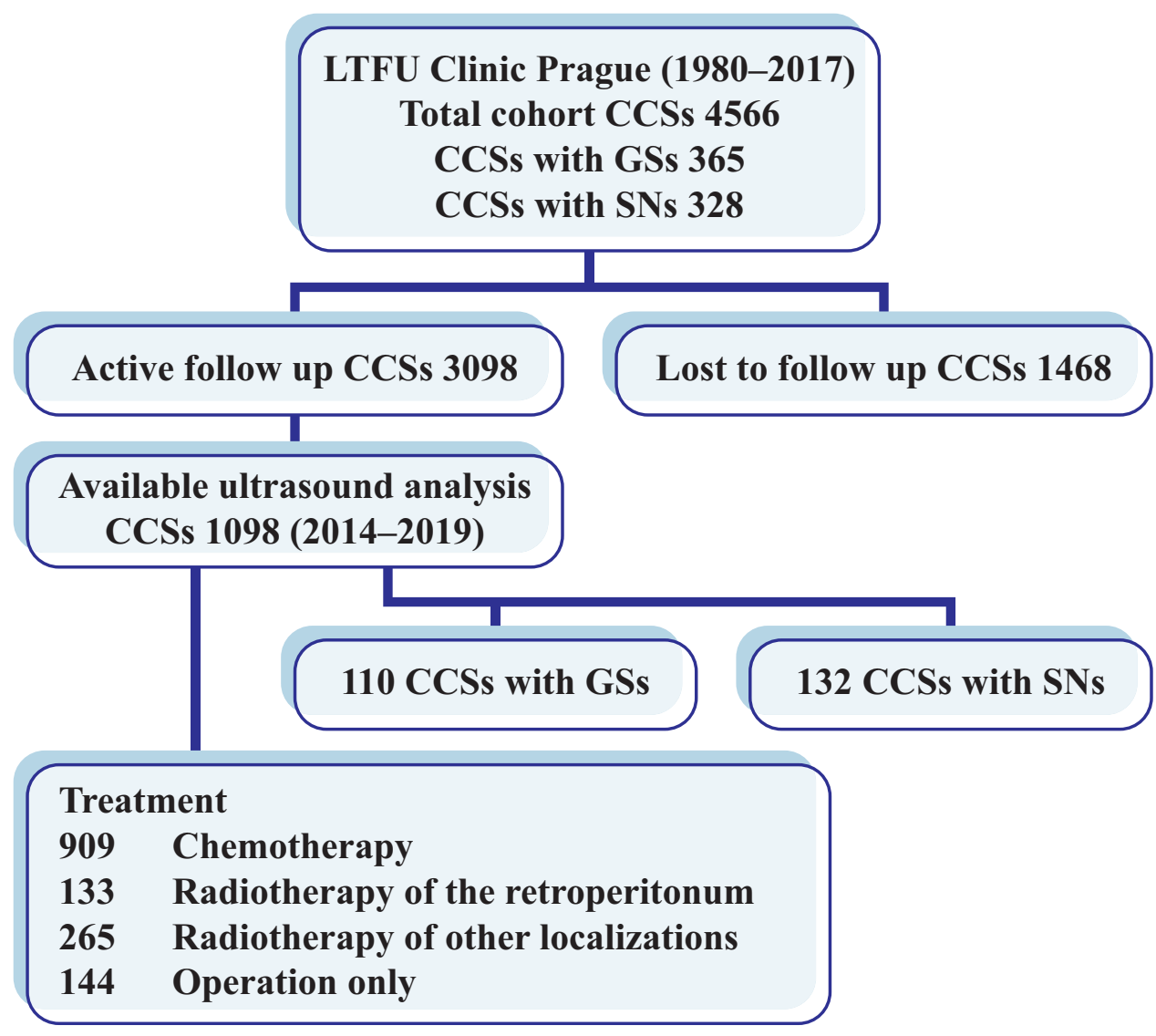

Figure I A summary of the cohort definition.

Abbreviations: LTFU, long-term follow-up; CCSs, childhood cancer survivors; GSs, genetic syndromes; SNs, subsequent neoplasms.

appearance of the lesion was determined by retrospectively evaluating prior ultrasounds of CCSs with SAML.

\section{Statistical Analysis}

The occurrence of SAMLs (simple prevalence and with respect to risk factors) was evaluated using survival analysis methods, ie Kaplan-Meier estimation of the proportion of SAML-free patients and the Cox proportional hazards model for estimation of hazard ratios. Simple frequencies observed in our sample are also presented. In the case of median time to SAML development, two estimation methods were used - survival analysis ("survival-median", based on a linear interpolation of the Kaplan-Meier curves stating the estimated time until half of the total population develops SAML) and the simple median of the observed times ("observed-median", stating the median time interval of those who actually developed SAML).

Statistical processing and analysis was performed in STATISTICA data analysis software system (StatSoft, Inc.2013, Version 12, www.statsoft.com).

\section{Ethical Considerations}

This study was conducted in accordance with the Declaration of Helsinki. Written informed consent was obtained from all study participants aged $\geq 18$ years, and from the parents/legal guardians of participants aged $<18$ years; they agreed that the data from their medical records would be used in the research. Ethical approval for this study was waived by the Ethics Committee of Motol University Hospital in view of the retrospective nature of the study, and because all the procedures being performed were part of routine care.

\section{Results}

SAMLs in CCSs - Ultrasound Findings, Prevalence, and Follow-Up

SAMLs were confirmed in 48 (4.4\%, $95 \%$ confidence interval (CI) 3.3-5.8\%) CCSs; 32 CCSs developed SAMLs during the study period, while 16 developed them before 2014 . The median number of ultrasound assessments with SAMLs was three (range 1-8), and the observed-median time 
Table I Characteristics of the Studied CCSs

\begin{tabular}{|c|c|c|c|c|c|c|c|}
\hline $\begin{array}{l}\text { Study Group } \\
\text { Characteristics }\end{array}$ & CCSs & $\begin{array}{l}\text { Median Age at Cancer dg } \\
\text { Years IQR }\end{array}$ & $\begin{array}{l}\text { Median Follow- Up } \\
\text { Years IQR }\end{array}$ & $\mathbf{F} / \mathbf{M}$ & GS $^{\mathbf{a}}(\%)$ & $\begin{array}{l}\text { SMNs } \\
(\%)\end{array}$ & $\begin{array}{l}\text { SBNs }{ }^{c} \\
(\%)\end{array}$ \\
\hline Total & 1098 & $4.3(1.4-11.4)$ & $8.5(5.1-14.2)$ & $525 / 573$ & $110(10)$ & $70(6.4)$ & $62(5.6)$ \\
\hline Bone tumors & 73 & $10.9(7.1-13.5)$ & $6.4(11.0-4.4)$ & $32 / 41$ & $3(4.1)$ & $2(2.7)$ & $7(10)$ \\
\hline CNS tumors & 61 & $5.2(2.9-9.2)$ & $12.7(8.9-\mid 7.1)$ & $31 / 30$ & $22(36)$ & $6(10)$ & $3(4.9)$ \\
\hline Germ cell tumors & 63 & $8.5(1.1-15.3)$ & I6.I (9.4-23.6) & $30 / 33$ & $4(6.4)$ & $2(3.2)$ & $2(3.2)$ \\
\hline Hodgkin lymphoma & 153 & $14.4(10.1-16.5)$ & $8.3(5.4-18.6)$ & $74 / 79$ & $7(4.6)$ & $25(16)$ & $17(11)$ \\
\hline Nephroblastoma & 144 & $3.1(1.5-4.4)$ & $12.3(7.2-16.4)$ & $85 / 59$ & $12(8.3)$ & $5(3.4)$ & $7(4.9)$ \\
\hline Neuroblastoma & 172 & $1.2(0.3-2.6)$ & $10.6(7.1-14.9)$ & $87 / 85$ & $8(4.6)$ & $9(5.2)$ & $6(3.5)$ \\
\hline $\begin{array}{l}\text { Non-Hodgkin } \\
\text { lymphoma }\end{array}$ & 116 & $8.3(5.0-12.8)$ & $8.2(5.4-12.8)$ & $37 / 79$ & 0 & $9(7.8)$ & $9(7.8)$ \\
\hline Retinoblastoma & 64 & $0.9(0.6-2.0)$ & $9.3(5.2-14.4)$ & $31 / 33$ & $28(44)$ & I (I.6) & 0 \\
\hline $\begin{array}{l}\text { Other malignant } \\
\text { tumors }^{\mathrm{d}}\end{array}$ & 133 & $2.0(0.9-8.8)$ & $7.4(3.7-13.2)$ & $59 / 74$ & $15(11.4)$ & $4(3.0)$ & $3(2.3)$ \\
\hline Soft tissue tumors & 119 & $5.8(2.0-1.9)$ & $7.8(4.5-12.6)$ & $59 / 60$ & II (9.3) & $7(5.9)$ & $8(6.7)$ \\
\hline
\end{tabular}

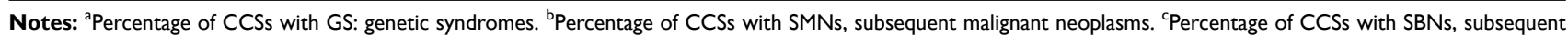
benign neoplasms. ${ }^{\mathrm{d} D i a g n o s i s:} 55$ histiocytosis; 32 hepatoblastoma; 25 various carcinoma; 9 melanoma; 12 other solid tumors $\leq 3$ per type.

Abbreviations: CCSs, childhood cancer survivors; IQR, interquartile range, ie lower-upper quartile; F, female; M, male.

between the first cancer dg and SAMLs was 22.62 years. The Kaplan-Meier estimate of the proportion of SAML-positive CCSs reached slightly beyond 50\% (Figure $2 \mathrm{~A}$ ). The survival-median time between the first cancer and the SAML diagnosis was 33.56 years, with an estimated 20 -year prevalence of $7.84 \%$ (95\% confidence interval (CI) $3.92-$ $11.75 \%)$, growing rapidly to $17.97 \%(11.07-24.86 \%)$ and $38.21 \%(27.49-48.93 \%)$ at 25 and 30 years after the first cancer, respectively. The median follow-up time was 8.52 years (Figure 2B), which is substantially shorter than the typical time for SAML development. However, owing to the large overall sample size, 116 CCSs at risk of SAMLs were still followed for at least 20 years. The median age at SAML occurrence was 27.91 years (Figure 2C). The Kaplan-Meier curve showing the age-related incidence of SAMLs is provided in (Figure 2D). Multiple ultrasound assessments were obtained in 40 CCSs over a median period of 3.8 years (range 3 to 173 months); 14 (35\%) cases of progression in size and nine (23\%) cases of progression in lesion number were described. The average size progression in the largest dimension was $4 \mathrm{~mm}$. None of these lesions was found to show any atypical features on subsequent ultrasound.

\section{SAMLs in CCSs - Association with Cancer Treatment Factors}

CCSs with SAMLs had the following cancer diagnoses: Hodgkin lymphoma, 11; neuroblastoma, 9; nephroblastoma, 6; non-Hodgkin lymphoma, 4; soft tissue sarcoma, 5; retinoblastoma, 5; germ cell tumors, 3; bone tumors, 3; and other malignant tumors, 2. GSs were recorded in seven (15\%) CCSs with SAMLs (Rb1 del., 4; LFS, 1; NF, 1; FAP, 1). The presence of GSs was not related to the number of observed SAML cases or to the risk of SAML development. Also, we did not observe any signs of association between the age at first cancer diagnosis and SAMLs (Figure 3A). The observed occurrence of SAMLs was higher in female, CCSs after chemotherapy, and CCSs after radiotherapy of the retroperitoneum (Table 2). A convincingly higher risk of SAML development was confirmed only for radiotherapy (1.65-fold higher with $95 \%$ CI $0.90-3.02$, Figure 3B). The effects of sex (1.60-fold higher risk for females, 95\% CI 0.84-3.03, Figure 3C) were seemingly similar, however while the survival curve of patients after radiotherapy starts separating from the rest of CCSs at ca. 13 years after primary 
A

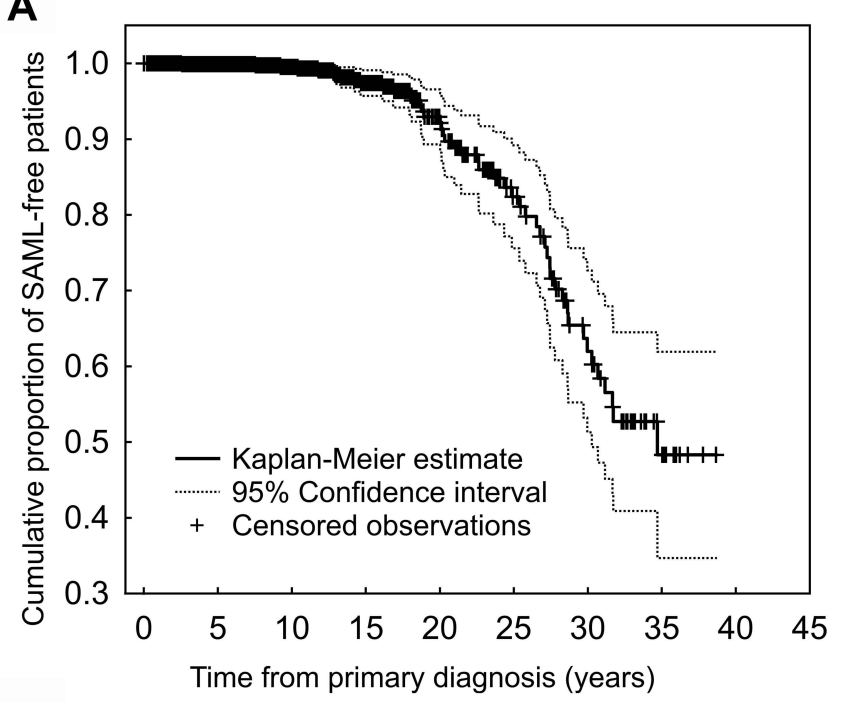

C

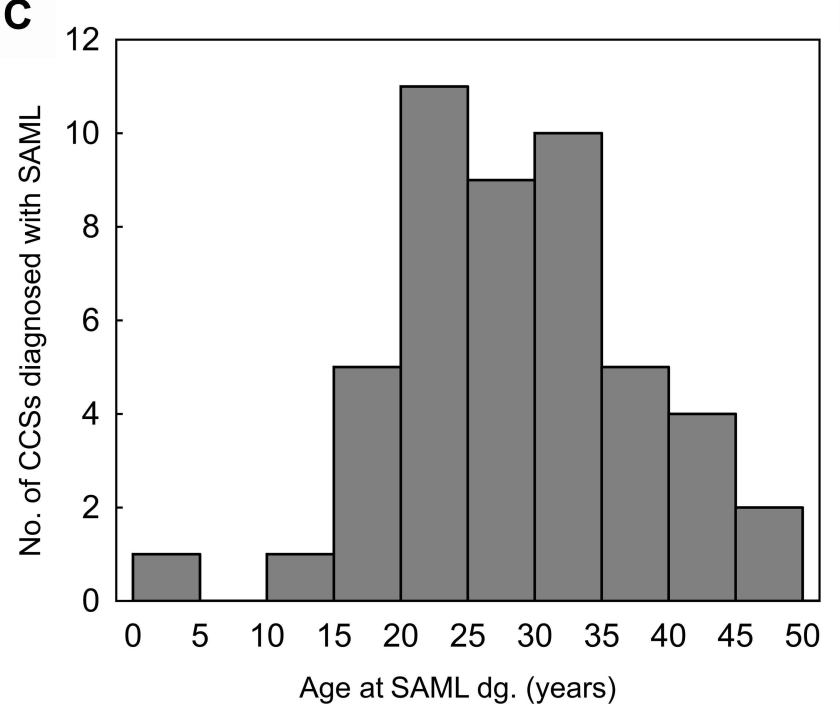

B

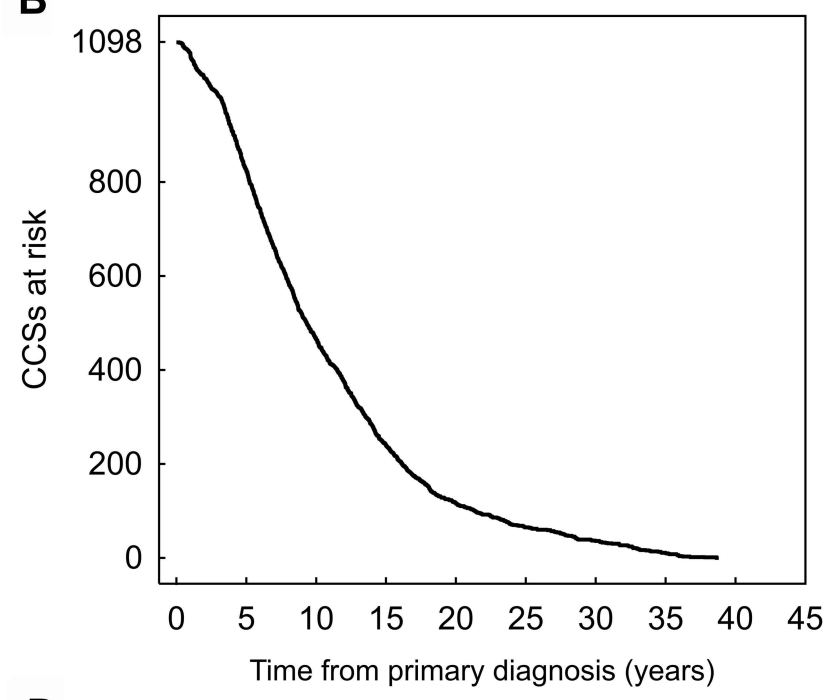

D

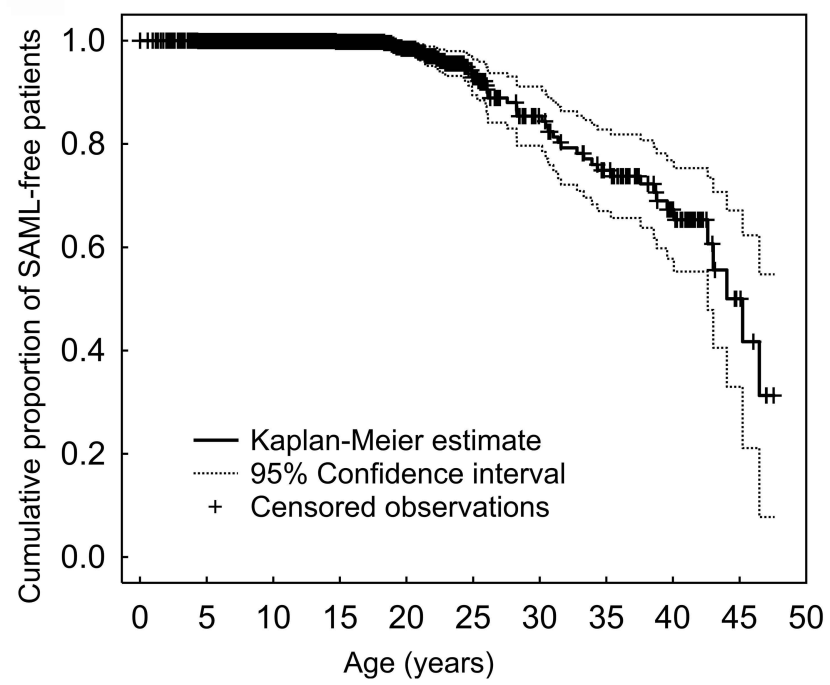

Figure 2 Summary of SAMLs, renal sporadic angiomyolipomas without histological confirmation, occurrence. Kaplan-Meier estimation of the proportion of SAML-free CCSs, childhood cancer survivors (A). Number of SAML-free CCSs remaining in the study at the given follow-up time (B). Histogram showing the distribution of age at SAML diagnosis (C). The Kaplan-Meier curve showing the age-related incidence of SAMLs (D).

diagnosis, for sex the separation does not come until after 20 years, when the number of patients in followup and hence the robustness of the observation is much lower. For chemotherapy, the risk of SAMLs was identical up to ca. 15 years of follow-up, after which there were only $12 \mathrm{CCSs}$ without previous chemotherapy left in observation, preventing any comparison beyond this point. In fact, the follow-up of both female and CCSs after chemotherapy was notably longer (1.33-fold with 95\% CI 1.17-1.50 for sex, and 1.70-fold with 95\% CI 1.44-14.99 for chemotherapy), thus explaining the differences in the observed numbers of SAML cases (Table 2).

\section{SAMLs in CCSs - the Correlation with SNs}

Out of 48 CCSs with SAMLs, 20 (42\%) had also been diagnosed with SNs. The presence of an SN appeared to be a risk factor of SAML development only in follow-ups exceeding 20 years (Figure 4A). The relative occurrence of SAMLs together with SNs (Table 2) was amplified even further by longer follow-up of CCSs with SNs in comparison to those without $\mathrm{SNs}$ (4.76-fold increase with a relatively narrow 95\% CI of 3.80-5.95, Figure 4B).

Twenty CCSs with SAMLs had 10 SMNs and 17 SBNs; the corresponding details are shown in Table 3. There were six SMNs and ten SBNs diagnosed before 

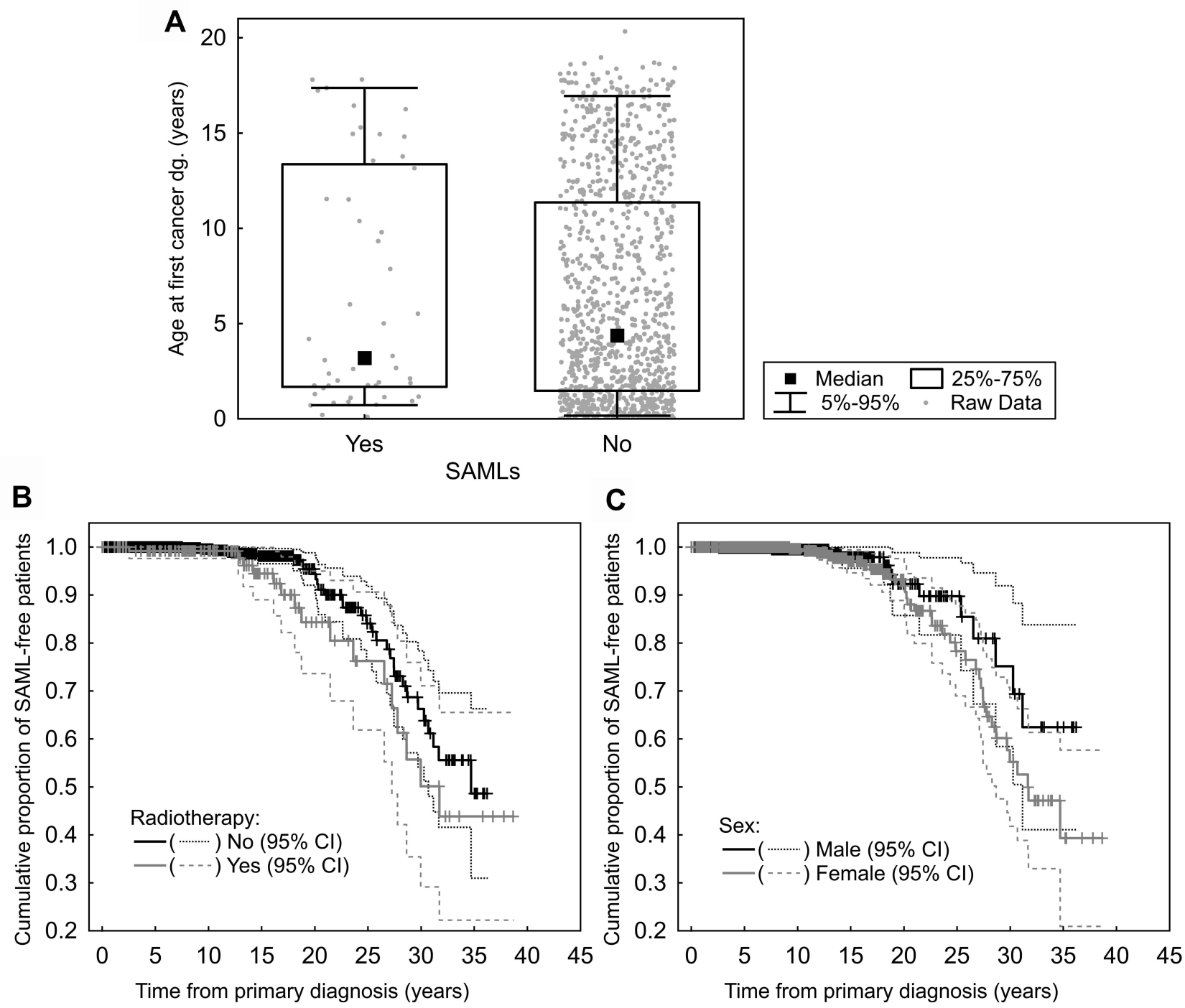

Figure 3 Comparison of age at first cancer diagnosis between CCSs, childhood cancer survivors with and without SAML, renal sporadic angiomyolipomas without histological confirmation (A); Kaplan-Meier plots of the risk of SAMLs in relation to radiotherapy of the retroperitoneum (B) and sex (C).

SAMLs, with a median time of 7.75 years (range 2 months-29 years), and two SMNs and six SBNs developed after the diagnosis of SAMLs, with a median time of 2.41 years (range 2-51 months). Two SMNs and one SBN were diagnosed on the same date as the SAMLs.

\section{Discussion}

The occurrence of SAMLs in CCSs and associations with cancer treatment factors have not yet been described. In our cohort, the occurrence of SAML was conditioned by the follow-up period - longer observation times provide a higher risk of of SAML development. Therefore, even though there were only $4.4 \%$ of SAML-positive CCSs in our cohort by simple numbers, the Kaplan-Meier estimate of SAML prevalence, which takes the effect of follow-up time into account, showed a much higher prevalence ( $\sim 40 \%$ after 30 years from the primary diagnosis). Even though the Kaplan-Meier estimate may be slightly exaggerated (for reasons that will be discussed further below), the fact that a majority of patients in our sample were not observed long enough to develop SAMLs makes the Kaplan-Meier estimation method much more suitable than estimation by simple frequencies.

The prevalence in the general population varies between $0.1 \%$ and $0.44 \% .{ }^{4,5,15}$ Fittschen et $\mathrm{al}^{5}$ in one the largest studies conducted so far, confirmed SAMLs in 270 out of 61,389 , ie $0.44 \%$ non-cancer patients who had retroperitoneal ultrasound assessment. Despite our smaller cohort, the occurrence of SAMLs in CCSs was conclusively higher, ie at least 10 times higher (according to the 
Table 2 Numbers of Observed SAML Lesions with Respect to Sex, Radiotherapy of the Retroperitoneum, GSs, and SNs

\begin{tabular}{|c|c|c|c|c|c|}
\hline & $\begin{array}{l}\text { No } \\
\text { SAMLs }\end{array}$ & $\begin{array}{l}\text { All } \\
\text { SAMLs }\end{array}$ & $\begin{array}{l}\text { Unilateral Single SAMLs } ; \mathbf{R} / \\
\mathbf{L}^{c}\end{array}$ & $\begin{array}{l}\text { Multiple Unilateral } \\
\text { SAMLs }\end{array}$ & $\begin{array}{l}\text { Bilateral } \\
\text { SAMLs }\end{array}$ \\
\hline All CCSs & 1050 & 48 & $27(56 \%) ; 13 / 14$ & II (23\%) & $10(21 \%)$ \\
\hline Female CCSs & $490(47 \%)$ & 35 (73\%) & - & - & - \\
\hline $\begin{array}{l}\text { CCSs after } \\
\text { chemotherapy }\end{array}$ & $862(82 \%)$ & 47 (98\%) & - & - & - \\
\hline CCSs after radiotherapy & 117 (II\%) & $16(33 \%)$ & $10(21 \%)$ & $4(8 \%)^{d}$ & $2(4 \%)$ \\
\hline CCSs with GSs & $103(10 \%)$ & $7(15 \%)$ & $2(4 \%)$ & $2(4 \%)$ & $3(6 \%)$ \\
\hline CCSs with SNs & 112 (II\%) & $20(42 \%)$ & II (23\%) & $7(15 \%)$ & $2(4 \%)$ \\
\hline
\end{tabular}

Notes: ${ }^{\text {P}}$ Percentages calculated from a total of 1050 non-SAML CCSs. ${ }^{b}$ Percentages calculated from a total of $48 \mathrm{CCSs}$ with SAMLs. ${ }^{\mathrm{R}} \mathrm{L} / \mathrm{L}=$ involvement of right/left kidney. d 3 out 4 CCSs with radiotherapy of the retroperitoneum had SNs.

Abbreviations: CCSs, childhood cancer survivors; SAMLs, renal sporadic angiomyolipomas without histological confirmation; GSs, genetic syndromes; SNs, subsequent neoplasms.

conservative and almost certainly underestimated observed frequency) than that in the general population. Moreover, the CCSs in our sample were substantially younger than the ages reported in other studies in "non-cancer populations" (median age, CCSs vs general population: 27.9 years vs $50-60$ years), ${ }^{1,3,5,7,14,16}$ which is another reason to expect the occurrence of SAML in CCSs to be in fact even higher after the CCSs reach a comparable age.

A majority of the previous analyses reported subcentimeter sizes of SAMLs, ${ }^{2,15}$ which is in agreement with our findings. The median size of SAMLs in CCSs was $8 \mathrm{~mm}$, similar to the findings published by Chan et al, ${ }^{15}$ where 217 patients had a median SAML size of $9 \mathrm{~mm}$. In our study, equal right and left kidney involvement was observed, which has also been previously reported for "non-cancer populations." "-3

Among the CCSs, $23 \%$ showed multiple lesions and $21 \%$ showed bilateral lesions. This contrasts with the general population where multiple and bilateral SAMLs are rare. ${ }^{2,3,5}$ In a cohort of 1493 non-cancer patients with SAMLs, Hussain et $\mathrm{al}^{2}$ observed only $11 \%$ with multiple and $6 \%$ with bilateral lesions. Our current observation does not support a simple direct effect of radiotherapy, as only $8 \%$ of the CCSs with multiple lesions and $2 \%$ of the
A

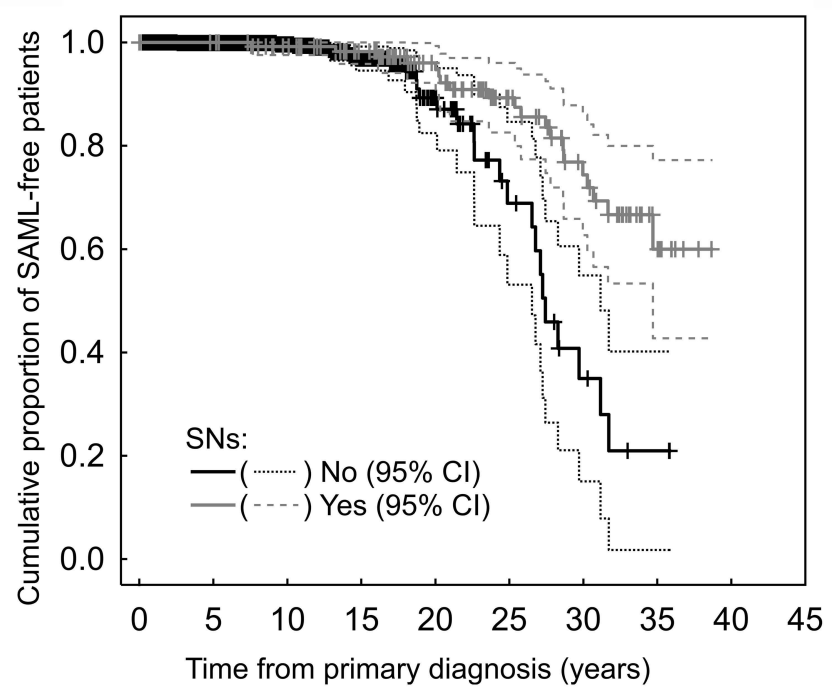

B

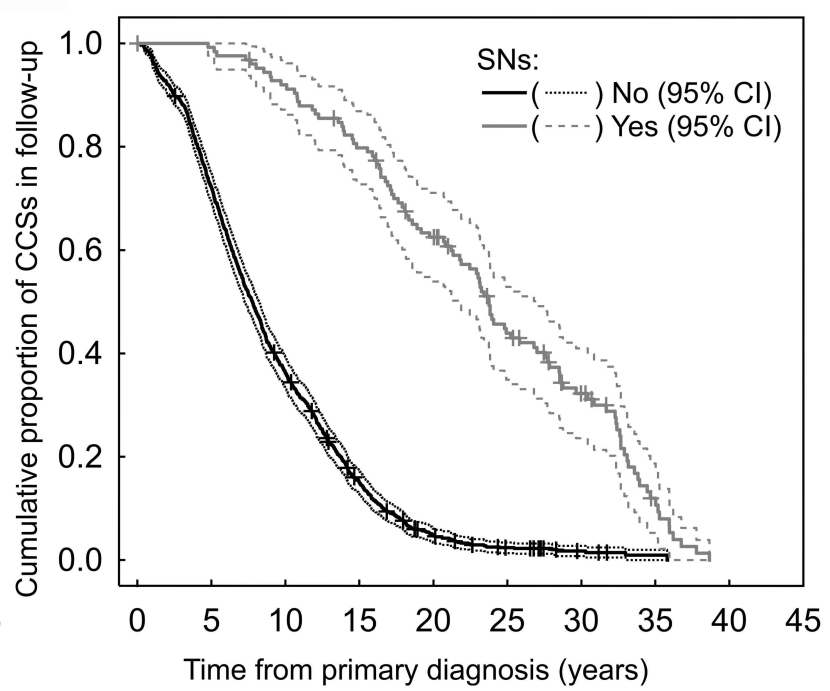

Figure 4 Development of SAMLs occurrence with respect to SNs, subsequent neoplasms- increased risk of SAML s in childhood cancer survivors with SNs was observed in patients with the longest follow-up (A). The follow-up of SNs-free CCSs in our study was substantially shorter than that of CCSs with SNs (B). 
Table 3 CCSs with SNs and SAMLs

\begin{tabular}{|c|c|c|c|c|c|c|c|}
\hline $\begin{array}{l}\text { Primary } \\
\text { Diagnosis }\end{array}$ & $\begin{array}{l}\text { Number of } \\
\text { CCSs }\end{array}$ & $\begin{array}{l}\text { Median Age at SAMLs dg } \\
\text { Years }\end{array}$ & $\begin{array}{l}\text { Median Follow-Up to SAMLs } \\
\text { dg Years }\end{array}$ & $\begin{array}{l}\mathbf{F} / \\
\mathbf{M}\end{array}$ & GS & SMNs ${ }^{\mathrm{a}}$ & SBNs ${ }^{b}$ \\
\hline Total & 20 & $34.6(19.1-47.5)$ & $27.3(6.6-34.4)$ & $\begin{array}{l}15 / \\
5\end{array}$ & 2 & 10 & 17 \\
\hline Germ cell tumors & 3 & $27.5(22.9-30.2)$ & $26.4(21.0-30.0)$ & $2 / 1$ & 0 & I & 2 \\
\hline Hodgkin lymphoma & 8 & $40.9(21.5-47.5)$ & $25.3(6.6-31.7)$ & $7 / 1$ & FAP & 2 & 7 \\
\hline Neuroblastoma & 4 & $30.3(19.1-42.7)$ & $28.5(18.1-32.9)$ & $1 / 3$ & 0 & 4 & 3 \\
\hline Nephroblastoma & 1 & 27.6 & 27.5 & $\mathrm{I} / 0$ & 0 & 0 & I \\
\hline $\begin{array}{l}\text { Non Hodgkin } \\
\text { lymphoma }\end{array}$ & 3 & $38.6(35.4-43.7)$ & $30.7(20.1-34.4)$ & $3 / 0$ & 0 & 2 & 4 \\
\hline Retinoblastoma & I & 26.1 & 25.4 & $1 / 0$ & $\begin{array}{l}\text { Rbl } \\
\text { el. }\end{array}$ & I & 0 \\
\hline
\end{tabular}

Notes: ${ }^{a}$ Carcinoma 5; CNS tumor 2; germ cell tumor I; neuroblastoma I; soft tissue sarcoma I. b Fibroepithelial tumor 8; myoma 4; non-melanoma skin cancer (NMSC) 2; osseus/chondro tumor 2; peripheral nerve sheath tumor I.

Abbreviations: CCSs, childhood cancer survivors; SAMLs, renal sporadic angiomyolipomas without histological confirmation; SNs, subsequent neoplasms.

CCSs with bilateral lesions developed their SAMLs in the field of radiotherapy. Moreover, three out of four CCSs with multiple SAMLs and radiotherapy of the retroperitoneum also developed SNs outside the radiotherapy field.

Based on our experience and other published reports, ${ }^{15,17}$ the ultrasound technique is limited with reference to the measurement of very small lesions. Consequently, the progression of size in the current study was defined as at least $3 \mathrm{~mm}$. Progression in size was observed in $14(35 \%)$ CCSs with SAMLs over a period of 44 months. Despite the similar size of SAMLs in CCSs, higher progression was observed than previously reported in "non-cancer" studies, where the most SAML did not grow. $2,7,15$

SAML risk factor assessment presents another important point regarding the importance of applying survival analysis methods. While simple frequencies are susceptible to a strong bias if the follow-up periods in the compared groups are not balanced, survival analysis methods take this factor into account. For instance, studies in noncancer populations observed female predominance; ${ }^{5-7}$ the possible effects of sex hormones were described. ${ }^{6}$ Even though simple proportions of female and male CCSs with SAMLs in our sample seem to confirm female sex as a risk factor, the survival-like analysis of the risk of SAML development showed some, but not very convincing effect.

The occurrence of angiomyolipomas at a young age was described only in TSC in several studies. ${ }^{1,3,4}$ Up to one-fifth of angiomyolipomas are associated with $\mathrm{TSC}^{2}$ these develop in ages between 3.8 and 23 years ${ }^{18}$ and are usually presented as multiple and bilateral lesions. Even though TSC was not observed in our study, the current characteristics of SAMLs in CCSs appear to have some features more similar to angiomyolipomas in TSC. The median age of SAMLs in CCSs was higher than that reported in TSC, but was much younger than in the general population. There were more multiple and bilateral lesions. In addition, the occurrence of other benign tumors is a part of this GS, ${ }^{10,18}$ as was observed in our CCSs with SAMLs.

Although the association with other types of GSs was not confirmed in this study, correlations between SAMLs and other GSs - MEN1, NF1, and LFS have been reported. ${ }^{12,13,19}$ These authors also assumed possible genetic links to the SAML development. The last two named GSs were also confirmed in CCSs with SAMLs in our study. We were not able to detect any relationships among these three antioncogenes (MEN1, NF1, and TP53) which are affected in the above-mentioned GS; relationships between these antioncogenes and the TSC2 and TSC1 genes were also not apparent. Further epidemiological and genetic studies will be needed to assess the relationship between GSs and the prevalence of SAMLs.

Studies in CCSs have clearly shown that radiotherapy increases the risk of various SNs. ${ }^{20-22}$ The current study also found that CCSs who received radiotherapy of the 
retroperitoneum had somewhat a higher risk of developing SAMLs. Although SAMLs cannot be termed as SBNs as the analysis was done only by ultrasound without histological confirmation, the time to SAML development since radiotherapy treatment was typical for radiotherapyinduced SNs. ${ }^{20}$

Some CCSs may experience multiple SNs with increasing age. ${ }^{20-22}$ The Childhood Cancer Survivors Study (CCSS), ${ }^{20}$ Dutch Childhood Oncology GroupLong-Term Effects After Childhood Cancer (DCOGLATER) Study, ${ }^{21}$ and also our previous study ${ }^{22}$ suggested that SBNs could be useful as risk markers for SMN development. In the current study, there was a high occurrence of SAMLs in CCSs with SNs, and 30\% of the SNs were diagnosed after SAMLs. The fact that the occurrence of SAMLs often coincided with SNs is not surprising given that detecting both these conditions is influenced by the patient's follow-up period, and they are thus likely to be observed at the same time. In our survival-like analysis, a higher risk was shown only later in the follow-up, which, while providing only limited confidence due to smaller number of observed CCSs, also represent the typical time of SAML's onset. It is important to note that in our study many CCSs with SNs did not participate in this study, as they had already been transferred to the adult oncology department or had succumbed to the disease.

There is no consensus regarding the surveillance of asymptomatic SAMLs. ${ }^{4,7,11}$ Hussain et $\mathrm{al}^{2}$ reported that small lesions $<3 \mathrm{~cm}$ could be disregarded. Chan et al ${ }^{15}$ suggested active surveillance for SAML $>2 \mathrm{~cm}$. Maclean et $\mathrm{al}^{17}$ suggested than only solitary SAMLs $<2 \mathrm{~cm}$ could be disregarded. As described above, CCSs with SAMLs may have some genetic susceptibility, and thus a higher progression of SAMLs and a higher occurrence of SNs were observed. For these reasons, continuous yearly surveillance of CCSs who develop SAMLs is important.

The limitation of our analysis is the relatively short follow-up of the included CCSs; consequently, data on the long-term prevalence of SAMLs were limited. Nevertheless, the survival analysis methods (ie KaplanMeier estimation) still allow for a relevant analysis of the data as long as a sufficient number of patients is followed for the period of time for which the prevalence estimate is made. However, Kaplan-Meier estimates may be slightly exaggerated as they are based predominantly on the oldest patients in the sample, for whom overall care was naturally slightly worse in comparison to that for CCSs with recent childhood cancers. This relatively recent diagnosis of the primary tumor was also the major reason for the short patient follow-up. However, in approximately $9 \%$ of the patients, the long-term abdominal ultrasound follow-up was intentionally terminated because of their relatively low-risk primary diagnosis. Consequently, the proportion of high-risk patients increased slightly during the followup, which likely marginally contributed to the exaggeration of our Kaplan-Meier estimates. Nevertheless, they are still more realistic than frequency-based observations, which are highly dependent on the follow-up period. As SAMLs and SNs are both relatively rare or delayed events in CCSs, to better understand the relationship between these two conditions, larger samples and/or longer followup periods will be necessary.

\section{Conclusion}

The present study observed that the occurrence of SAMLs in CCSs was at least 10 times higher than that reported in non-cancer studies. Compared to the general population, CCSs with SAMLs were younger, there was only marginal female predominance, and more bilateral and multiple SAML lesions were detected. SAML in CCSs appears to be more similar in some respects (younger age at diagnosis, more frequent multiple or bilateral lesions) to angiomyolipomas associated with TSC than to sporadic in "non-cancer population". Moreover, coincidence between SAMLs and SNs was observed. Our results support the hypothesis that the development of SAML in CCSs is not only a late effect of therapy, but also other factors may be expected.

\section{Abbreviations}

BWS, Beckwith-Wiedemann syndrome; CCSs, childhood cancer survivors; CCSS, The Childhood Cancer Survivors Study; DCOG-LATER, Dutch Childhood Oncology Group-Long-Term Effects After Childhood Cancer; FAP, familial adenomatous polyposis; GS, genetic syndrome; IQR, interquartile range; LFS, Li-Fraumeni syndrome; MEN1, multiple endocrine neoplasia syndrome type 1; MLES, Motol Late Effect Studies; NF1, neurofibromatosis type 1; SAML, renal sporadic angiomyolipomas without histological confirmation; SBN, subsequent benign neoplasm; SMN, subsequent malignant neoplasm; SNs, subsequent neoplasms; Rb1-del., Rb-1 deletion; TSC, tuberous sclerosis complex. 


\section{Author Contributions}

All authors made substantial contributions to conception and design, acquisition of data, or analysis and interpretation of data; took part in drafting the article or revising it critically for important intellectual content; agreed to submit to the current journal; gave final approval of the version to be published; and agreed to be accountable for all aspects of the work.

\section{Funding}

This work was supported by the Czech health research council under Grant NV19-03-00245; and by the project "Center of Clinical and Experimental Liver Surgery" under Grant UNCE/MED/006.

\section{Disclosure}

The authors report no conflicts of interest in this work.

\section{References}

1. Lee KH, Tsai HY, Kao YT, et al. Clinical behavior and management of three types of renal angiomyolipomas. J Formos Med Assoc. 2019;118:162-169. doi:10.1016/j.jfma.2018.02.012

2. Hussain T, Lam V, Farhad M, et al. Can subcentimetre ultrasound detected angiomyolipomas be safely disregarded? Clin Radiol. 2020;75(4):287-292. doi:10.1016/j.crad.2019.12.006

3. Swärd J, Henrikson O, Lyrdal D, Peeker R, Lundstam S. Renal angiomyolipoma-patient characteristics and treatment with focus on active surveillance. Scand J Urol. 2020;54(2):141-146. doi:10.1080/ 21681805.2020.1716066

4. Flum AS, Hamoui N, Said MA, et al. Update on the diagnosis and management of renal angiomyolipoma. $J$ Urol. 2016;195(1):834-846. doi:10.1016/j.juro.2015.07.126

5. Fitttschen A, Wendlik IN, Oeztuerk S, et al. Prevalence of sporadic renal angiomyolipoma: a retrospective analysis of 61389 in - and out patients. Abdom Imaging. 2014;39:1009-1013. doi:10.1007/s00261014-0129-6

6. Boorjian SA, Sheinin Y, Crispen PL, et al. Hormone receptor expression in renal angiomyolipoma: clinicopathologic correlation. Urology. 2008;72(4):927-932. doi:10.1016/j.urology.2008.01.067

7. Fernández-Pello S, Hora M, Kuusk T, et al. Management of Sporadic renal angiomyolipoma: a systematic review of available evidence to guide recommendations from the European association of urology renal cell carcinoma guidelines panel. Eur Urol Oncol. 2020;3 (1):57-72. doi:10.1016/j.euo.2019.04.005

8. Giannikou K, Malinowska IA, Pugh TJ, et al. Whole exome sequencing identifies TSC1/TSC2 biallelic loss as the primary and sufficient driver event for renal angiomyolipoma development. PLoS Genet. 2016;12(8):e1006242. doi:10.1371/journal.pgen.1006242
9. Caliò A, Brunelli M, Segala D, et al. Angiomyolipoma of the kidney: from simple hamartoma to complex tumour. Pathology. 2021;53 (1):129-140. doi:10.1016/j.pathol.2020.08.008

10. Henske EP, Neumann HP, Scheithauer BW, et al. Loss of heterozygosity in the tuberous sclerosis (TSC2) region of chromosome band 16 p13 occurs in sporadic as well as TSC-associated renal angiomyolipomas. Genes Chrom Cancer. 1995;13(4):295-298. doi:10.1002/gcc.2870130411

11. Jinzaki M, Silverman SG, Akita H, Nagashima Y, Mikami S, Oya M. Renal angiomyolipoma: a radiological classification and update on recent developments in diagnosis and management. Abdom Imaging. 2014;39(3):588-604. doi:10.1007/s00261-014-0083-3

12. Neofytou K, Famularo S, Khan AZ. PEComa in a young patient with known Li-Fraumeni syndrome. Case Rep Med. 2015;2015:906981. doi:10.1155/2015/906981

13. Stone NN, Atlas I, Kim US, Kwan D, Leventhal I, Waxman JS. Renal angiomyolipoma associated with neurofibromatosis and primary carcinoid of mesentery. Urology. 1993;41(1):66-71. doi:10.1016/00904295(93)90249-A

14. Jain K, Das M, Chatterjee U, Datta C. Cytological features of a renal angiomyolipoma in a child-a case report. Diagn Cytopathol. 2019;47 (11):1190-1193. doi:10.1002/dc.24276

15. Chan KE, Chedgy ECP, Bent CL, Turner KJ. Surveillance imaging for sporadic renal angiomyolipoma less than $40 \mathrm{~mm}$ : lessons learnt and recommendations from the experience of a large district general hospital. Ann R Coll Surg Engl. 2018;100(6):480-484. doi:10.1308/ rcsann.2018.0040

16. Çalışkan S, Gümrükçü G, Özsoy E, Topaktas R, Öztürk Mİ. Renal angiomyolipoma. Rev Assoc Med Bras. 2019;65(7):977-981. doi:10.1590/1806-9282.65.7.977

17. Maclean DFW, Sultana R, Radwan R, McKnight L, Khastgir J. Is the follow-up of small renal angiomyolipomas a necessary precaution? Clin Radiol. 2014;69(8):822-826. doi:10.1016/j.crad.2014.03.016

18. Lam HC, Siroky BJ, Henske EP. Renal disease in tuberous sclerosis complex: pathogenesis and therapy. Nat Rev Nephrol. 2018;14 (11):704-716. doi:10.1038/s41581-018-0059-6

19. Dong Q, Debelenko LV, Chandrasekharappa SC, et al. Loss of heterozygosity at 11q13: analysis of pituitary tumors, lung carcinoids, lipomas, and other uncommon tumors in subjects with familial multiple endocrine neoplasia type 1. J Clin Endocrinol Metab. 1997;82 (5):1416-1420. doi:10.1210/jcem.82.5.3944

20. Armstrong GT, Liu W, Leisenring W, et al. Occurrence of multiple subsequent neoplasms in long-term survivors of childhood Cancer: a report from the childhood cancer survivor study. J Clin Oncol. 2011;29(22):3056-3064. doi:10.1200/JCO.2011.34.6585

21. Kok JL, Teepen JC, Van der pal HJ, et al. Incidence of and risk factors for histologically confirmed solid benign tumors among long-term survivors of childhood cancer. JAMA Oncol. 2019;5 (5):671-680. doi:10.1001/jamaoncol.2018.6862

22. Zichová A, Eckschlager T, Ganevová M, Malinová B, Lukš A, Kruseová J. Subsequent neoplasms in childhood cancer survivors. Cancer Epidemiol. 2020;68:101779. doi:10.1016/j. canep.2020.101779
Clinical Epidemiology

\section{Publish your work in this journal}

Clinical Epidemiology is an international, peer-reviewed, open access, online journal focusing on disease and drug epidemiology, identification of risk factors and screening procedures to develop optimal preventative initiatives and programs. Specific topics include: diagnosis, prognosis, treatment, screening, prevention, risk factor modification, systematic reviews, risk \& safety of medical interventions, epidemiology \& biostatistical methods, and evaluation of guidelines, translational medicine, health policies \& economic evaluations. The manuscript management system is completely online and includes a very quick and fair peer-review system, which is all easy to use. 